system, for which I have given the decoct. dulcam. with good effect, keeping the bowels open by means of the following mixture :-

R. Pulv. Rhei. Pulv. Carb. Magnes. āà 3 ss. Spt. Annis. gtt. xxx. Aquæ Cinnam. 3 vij. M. ft. Mistura. Cap. Coch. ij. Mag. ter in die.

If the consecutive fever ran high, with strong pulse, I lave taken from six to ten ounces of blood, and given a mixture of the Carbonate of Soda in Aqua Cinnam. But in general, the great and profuse perspiration relieves the whole system; and except debility, and sometimes dyspepsia, the rest of the treatment consists in relieving any symptom which may arise from confinement, \&c.

A table which follows the above, consists of a minute account of 24 patients, with their names, residences, \&c. who were treated as described by $\mathrm{Mr}$. B. The success is remarkable if the cases were severe, on which very important point the author is, unhappily, silent. It is stated, however, that, with the exception of two individuals, none found it necessary to repeat the dose more than three times, and most of them but once.

\title{
ANEURISM OF THE CAROTID ARTERY.
}

\section{Wound of the Carotid Artery, Followed by Aneurism, and Cured by} Ligature. By Edw. Fras. Dehane, M.R.C.S., of Eng.

Louisa Newelt, a delicate girl, ten years of age, on the 4 th January last, in the act of going down stairs with a dish in her hand, slipped down, and in her fall broke the dish, a sharp point of which punctured the neck. On my arrival, a few minutes after the accident, I found the little girl bleeding profusely from a wound about an inch above the clavicle, in the course of the carotid artery on the right side. About two pounds of blood had been already lost, and she appeared rapidly falling into a state of syncope-her lips being pallid, her ska cold and clammy, and the pulse at the wrist not to be felt. The wound in the neck might be large enough to admit the end of the finger.

Being doubtful whether the patient would revive, I immediately applied a compress of lint over the wound, and caused it to be retained there by pressure, and, in the meantime, prepared ligatures, \&c., to secure the bleeding vessel, but was surprised, upon its removal, to find that there was no hæmorrhage, notwithstanding she had very much revived from the state of syncope she had fallen into. I however waited some time, under the expectation of its renewal, but which did not follow, owing, as I suppose, to the obliquity of the puncture. I therefore secured the compress, and, having placed my patient in bed, left her. : It was not: until the fifth day afterwards that I removed the compress, when I found that the external wound had healed, but perceived a small pulsating tumor a little above the puncture. I desired the child to be kept quiet, and continued the pressure, both upon and below the tumor, as tight as it could be borne; it, notwithstanding, gradually increased, but not so 
much as to be very perceptible, till the night of the 19th, when it suddenly became enlarged to the size of a walnut, pulsating under the sterno cleido mastoideus muscle, and extending beyond it, and evidently in the line of the carotid-upon pressing which, all pulsation in the tumor ceased. At my visit on the following morning, I proposed to take up the vessel, which was immediately assented to by the parents of my little patient.

Having requested the attendance of my brother, Dr. Dehane, and Mr. Thompson, surgeon of this town, they agreed with me in opinion that no further time should be lost in performing the operation.

The patient being placed on her back, with her head inclining over the left shoulder, I commenced an incision from the base of the tumor, following the course of the sterno mastoideus muscle along its inner edge down to the clavicle. More difficulty was experienced in getting at the vessel than I had anticipated-the space between the base of the aneurism and bone not exceeding one inch, which small space was crossed by the thyroid veins, and moreover the depth of the cellular membrane was considerable, so that I could barely feel the vessel, with the point of my finger, by forcibly pushing back the sterno mastoideus muscle. I therefore found it necessary to extend the incision in a direction upwards and backwards, somewhat above the tumor; by doing which, I was at length enabled to get a sufficient space to pass my ligature round the vessel. This, however, I was unable to effect with the common aneurismal needle, but succeeded with an eyed probe, which I bent to an acute angle, and passed under the artery, armed with a single silk ligature; this being drawn tight, I had the satisfaction to find that all pulsation stopped in the tumor. The operation was thus concluded, without any further loss of blood than the trifling quantity which followed the first incision. I placed my patient in bed, and, having exhibited an anodyne, left her. At my visit in the evening, I found her tranquil, and free from pain; the right side of the face, which had become cold on tying the ligature, had resumed the natural temperature, and the pulse at the wrist was regular.

January 21st, 9, A. M.-Patient has slept well, but complains of a little thirst ; pulse 125 ; skin rather warmer than natural; no pulsation in the tumor. At 10, P. M., has had more refreshing sleep; tongue slightly furred.-A saline aperient given, but has not acted; to be continued 3tis horis.

22d.-Slept well; skin more cool; pulse 125 ; bowels have not yet acted ; wound lnoking well.-To take Ol. Ricini, 3 ss. 2dis horis.

23d.-Bowels have acted-has had two motions; skin cool; pulse 120 ; tongue less furred; has passed a good night.

24th. - Slept well; pulse 110 ; wound uniting at upper part ; slight watery discharge from the inferior part.-Cont. Mist. Salin. Aper.

25th. - Going on well. From this time to the 31 st, when the ligature came away, no bad symptom had occurred. Pulse is now 90 ; discharge more healthy, and much less in quantity; wound nearly closed, and the aneurism diminished to about the size of a Spanish nut. About a fortnight after the last report the wound had quite healed, and the aneurismal tumor scarcely perceptible.-Medical Gazetle. 